\title{
Removing Raindrops in Continuous Video Images for Fixed- Object Surveillance Systems
}

\author{
Nam-Bong Ha and Namgi Kim ${ }^{1}$ \\ Department of Computer Science, Kyonggi University \\ \{nbha,ngkim\}@kgu.ac.kr
}

\begin{abstract}
In this paper, a dynamic algorithm is proposed that is aimed at removing raindrops within fixed background video images according to the volume of raindrops. In surveillance videos with fixed backgrounds, when the field of vision is obstructed because of falling rain or snow, obtaining picture information on the objects becomes difficult. Therefore, obstacles such as raindrops that obstruct the fiela of vision tould be removed from pictures to obtain more specific picture information. The proposed technique obtains images on a periodical basis from a fixed background picture and assesses whether there are raindrops in the present screen among the obtained images. The proposed technique predicts the volume of raindrops by comparing images obtained periodically and removes raindrops by applying a different dynami algorithm based on the predicted volume of raindrops. This study embodied and experimented with the proposed technique and verified that the result was excellent.
\end{abstract}

Keywords: computer vision, rdin detection, ran removal, video surveillance

\section{Introduction}

In modern society olosed-circuif televisions and Internet protocol cameras are used for monitoring the occurnence of signficant abnormalities and damages by strengthening indoor and oatdoonsecurities on by photographing specific topographies, natural features, or interesting areas. Sach a technique helps view pictures photographed by cameras anywhere and anytime hrough networks and can monitor interesting areas regardless of time and place [1].

Several studies have been conducted on monitoring techniques that determine the conditions of objects through pictures obtained through surveillance cameras. There are several types of monitoring technologies including those used for registering specific objects and examining their changes, sensing changes in an area, and removing elements that obstruct monitoring. The technology that registers specific interesting objects and examines their changes is used to register cars, persons, and other objects of interest and detemmine their moving routes or behaviors. The technologies of sensing changes in monitored areas are used for preparing for damages due to cracks in specific topographies or natural features, as shown in Figure 1. For example, monitoring waterside structures or bridges and observe changes to determine the amount of damage occurred. In addition, a representative technology used for removing obstacles in area monitoring induces the removal of fog when it hinders the procurement of important information in the monitored area, as shown in Figure 2 [2-4,8].

This paper proposes a technology for removing raindrops that obstruct the monitoring of a specific object [5-7]. Currently, the technologies used for removing raindrops from video images extract raindrop candidates through color differences between prior and

${ }^{1}$ Corresponding author: Namgi Kim 
present images and by using the size and angle of raindrops. Some other technologies use pixels at locations in previous pictures and replace them at the same location of raindrops in the present picture because raindrops move downward over time. When the volume of raindrops is small, replacement with such a method is possible. Further, when the speed of rainfall is slow, the interval of photographing is important; however, raindrops may not be obviously removed according to the intervals. When the volume of raindrops is large, the pixels intended to replace may still be those of raindrops. Therefore, determining the interval of photographing video images and using a dynamic algorithm to remove raindrops according to their volume may lead to better results.

In this study, the candidate raindrops are determined and removed by using information on existing background colors by photographing fixed background videos. In addition, the volume of raindrops is predicted by repeating extraction process of candidate raindrops on a real time basis and obvious pictures are obtained using the dynamic raindrop remorval algorithm according to the volume of raindrops.
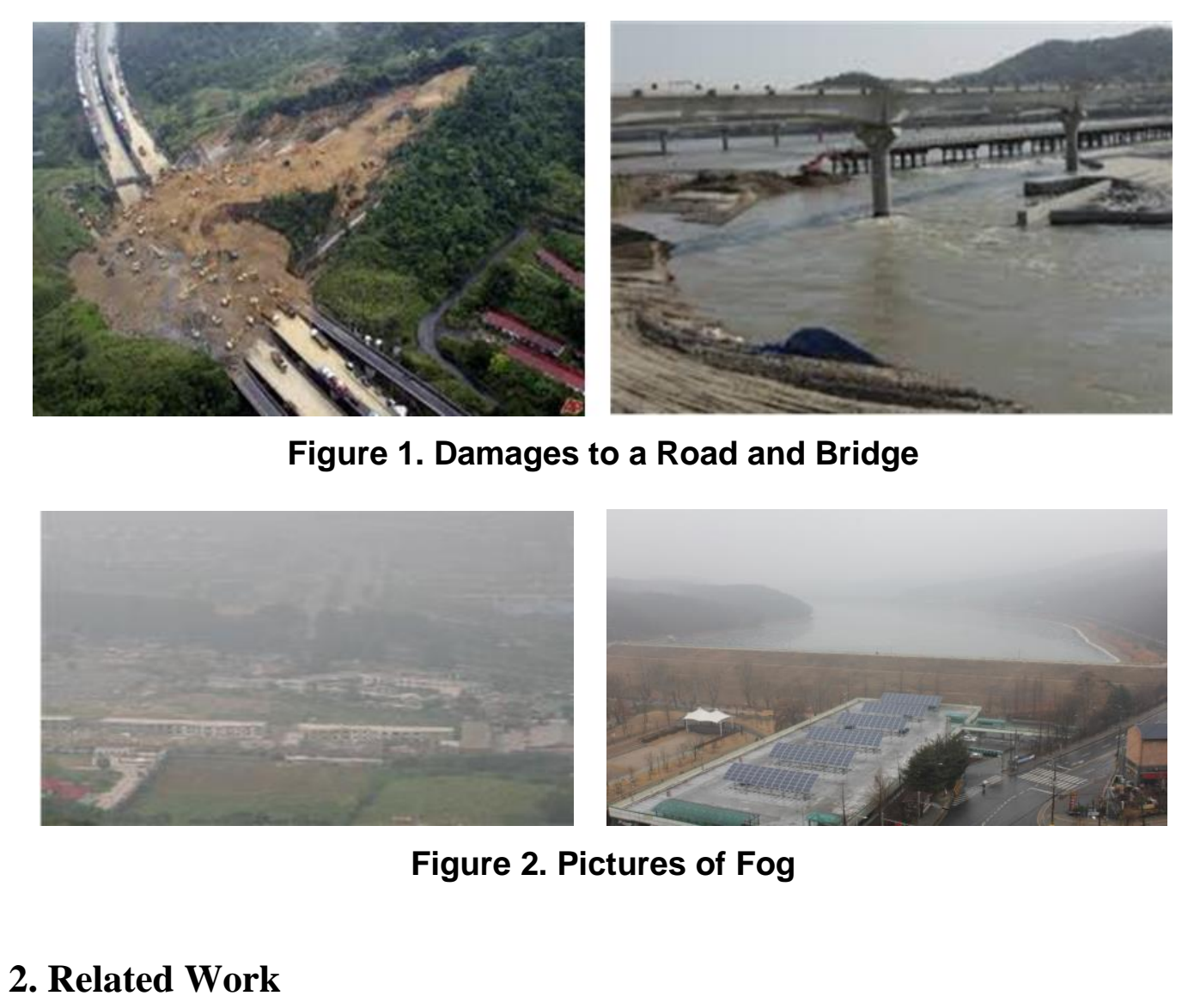

Figure 2. Pictures of Fog

All printed material, including text, illustrations, and charts, must be kept within the parameters of the $815 / 16$-inch (53.75 picas) column length and 5 15/16-inch (36 picas) column width. Please do not write or print outside of the column parameters. Margins are $3.3 \mathrm{~cm}$ on the left side, $3.65 \mathrm{~cm}$ on the right, $2.03 \mathrm{~cm}$ on the top, and $3.05 \mathrm{~cm}$ on the bottom. Paper orientation in all pages should be in portrait style.

\subsection{Picture Processing Equipment and Methods to Remove Rain from Pictures}

The method to detect and remove rain in pictures involves the removing of candidate raindrops according to their size, angle, and other characteristics. The pixels of a specific image and its earlier version are compared, and when the values of red, green, and blue (RGB) have crossed a certain threshold, they are considered as candidate raindrops. To 
extract false candidate raindrops from among all the candidate raindrops, the raindrop size and angle filtering that extract false candidate raindrops according to their size and angle respectively are used. Through such filtering, the final candidate raindrops are obtained. These are removed by bringing and replacing pixels of the relevant area form prior pictures without raindrops.

\subsection{Rain Removal in Videos by Using Kalman Filter [9]}

In [9], an algorithm was proposed for removing raindrops by using a Kalman filter. Although the brightness of the pixels affected by raindrops increases, they have color information of the original pixels. Therefore, the original pixels are restored by lowering the brightness of the rain-affected pixels. In this method, the recursive data is processed and the brightness value of each pixel may be measured. The advantages of this method include simple processing and using a formula to measure the average brightness repetitively in real time. However, because of unsuitable estimated mean yalue, recursive data processing needs to be periodically reset. In addition, if unsuitable estingated mean values are determined, the scenes in which a small amount of rain has been photographed and have small weights are selected, and a new mean value is determined.

\subsection{Detection and Removal of Rain from Videos [3]}

Grag and Nayar proposed an algorithm in which the characteristics of rain are considered and the raindrops within the pictures are removed The rains appearing in video images is expressed by a combmation of its dynamic characteristics and environmental photometry. Therefore, Grag and Nayar [3] presented a comprehensive analysis of visual effects of rain in images and developed a correlation model. The correlation model explains dynatmics of rain and a physics-based motion blur model. Based on the correlation model, an efficient algorithm for detecting and removing rain from videos was proposed

\subsection{Rain Removal in Videos by Combining Temporal and Chromatic Properties [10]}

Zhang and Li proposed a method in which visual and color characteristics of rain within picturesare allinfegrated, and a new rain removal algorithm is used. Visual characteristics refer to the characteristics of rain failing to cover entire pictures and color characteristics refer to the RGB values of pixels changed by rain. This technique finds, and removes raindrops in static or dynamic pictures through these two characteristios. The algorithm proposed by Zhang and Li may be applied to both moderate and heavy rains.

\section{Proposed Algorithm}

The algorithm proposed in this paper is divided into three parts. In the first part, the interval of obtaining images is determined. The locations of raindrops differ according to intervals, and the intervals of obtaining images play an important role. In the second part, candidate raindrops are extracted using colors in the fixed background. The volume of raindrops is predicted considering the rates of extracted candidate raindrops on the screen. In the final part, raindrops are removed according to the volume of the estimated raindrops by using a dynamic algorithm. 

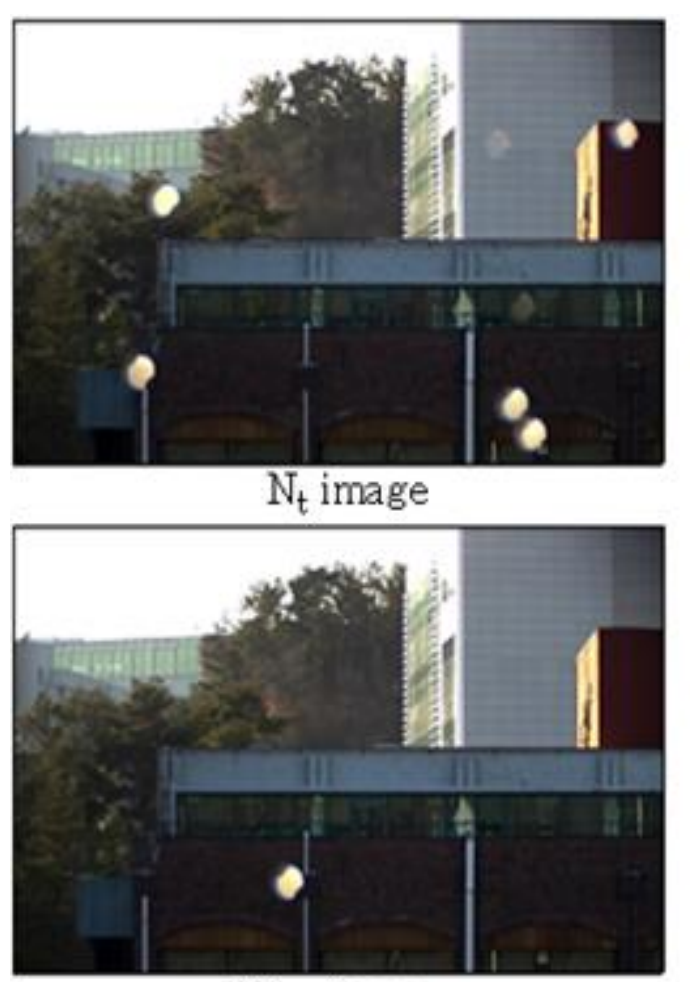

$\mathrm{N}_{t 2}$ image

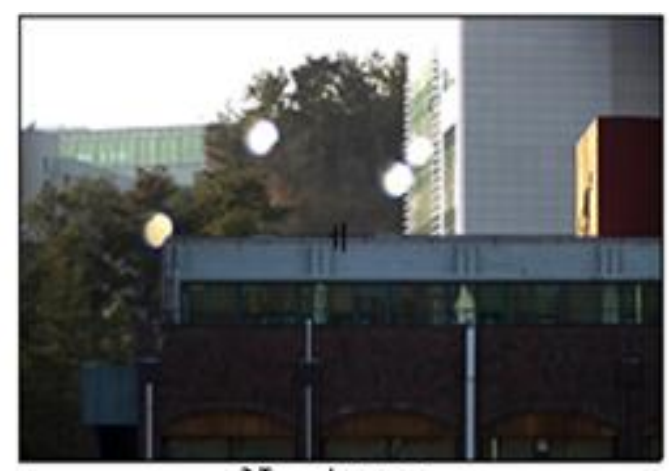

$\mathrm{N}_{\mathrm{t}-1}$ image

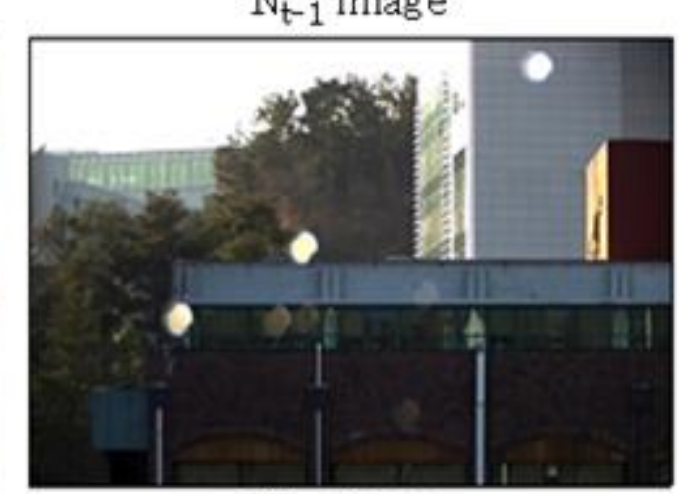

$\mathrm{N}_{\mathrm{t}-3}$ image

Figure 3. Pictures Obtained at á Certain Interval

\subsection{Picture Photographing Interval}

The algorithm proposed in this paper obtains images from surveillance videos at a certain interval. The images obtaimed at a certain interval are compared with the preceding images. The reasonfor comparison, as explained in Section 3.2, is that they are used for extracting candidate raindops and measuring the volume of raindrops. The interval of obtaining images from videos plays a very important role in the algorithm proposed in this paper. Raindrops move in the downward direction over time, and therefore raindrops in the images obtained from pictures will move downward over time. The key to the algorithm is replacing the pixels including raindrops with those without raindrops by comparing pixels in which raindrops are located in the current images with the pixels in the same Iocation in previous images. Therefore, when the interval of obtaining images from pictyres is too small, the pixels where raindrops are located overlap and the result is not good. Further, when the interval of obtaining images from videos is large, the locations of raindrops in continuous images will be sporadic. Thus, the interval of obtaining raindrops from videos is very important. In Figure 3, images are obtained at a certain interval.

\subsection{Extraction of Candidate Raindrops}

Raindrops increase the brightness of a photograph's background. When pixels in the relevant location are compared between versions of the same picture with and without raindrops, brightness is observed to have increased for the picture with raindrops. Therefore, to extract raindrops, brightness of the current image must be compared with a prior image and the pixels whose brightness has been heightened must be extracted. However, passing objects or small changes in light, may be wrongly extracted as raindrops. To avoid such wrongly extracted raindrops, the threshold value and the color of the background are utilized. The relevant video image shows a fixed background, and 
therefore its color information can be known. Pixels are usually composed of RGB color models. An RGB color model expresses colors in a mixture of red, green, and blue. The model has information on colors only, and is not appropriate to embody a computer vision algorithm. Therefore, it should be converted into a color model in which brightness elements are included. A hue, saturation, and value (HSV) model is a color model that expresses colors into hue, saturation, and value. Therefore, by converting the RGB color model into its corresponding HSV color model and utilizing the $V$ value, the brightness of the current and prior images are compared.

$$
\begin{gathered}
\mathrm{V} \leftarrow \max (\mathrm{R}, \mathrm{G}, \mathrm{B}) \\
\mathrm{S} \leftarrow\left\{\begin{array}{l}
\frac{V-\min (R \cdot G \cdot B)}{V} \text { if } V \neq 0 \\
0 \quad \text { otherwise }
\end{array}\right. \\
\mathrm{H} \leftarrow\left\{\begin{array}{l}
\frac{60(G-B)}{V-\min (R, G, B)} \\
120+\frac{60(B-R)}{V-\min (R \cdot G \cdot B)} \\
240+\frac{60(R-G)}{V-\min (R, G, B)} \text { if } V=R
\end{array}\right)
\end{gathered}
$$

As shown in equation (1), when RGB color model iscconverted into the HSV color model, $V$ becomes the largest value among the R, G, and B elements of the relevant pixel. As mentioned earlier, raindrops increase the brightness of the relevant background, and therefore the relevant pixel is considered a raindrop if the largest $V$ value is the same for the prior and current images. In addition, the largest element among $\mathrm{R}, \mathrm{G}$, and $\mathrm{B}$ values of the increased pixel and that of the relevant pixel with no raindrops must be the same for the existing known backgound. Figure 4 shows the flowchart for extracting candidate raindrops. First, the current and prior images are converted into the HSV color model from their corresponding RGB color model. By comparing the $V$ values of the current and prior images, the part in which the $V$ value has increased is extracted. Further, from the extracted $V$ yalues, those larget than $V_{\text {threshold }}$ are extracted. Here, $V_{\text {threshold }}$ is a value used to filter out the extracted part resulting from small changes in light. After applying the threshold value, the largest element among the R, G, and B values of the relevant pixel in the previous and presentbackground images are compared, and if the largest elements are the same, they areconsidered as raindrops, and candidate raindrops are thus extracted. Figure 5 shows candídate raindrops extracted through the relevant algorithm. The volume of raindrops dan be predicted through the amount of extracted candidate raindrops in the screen. 


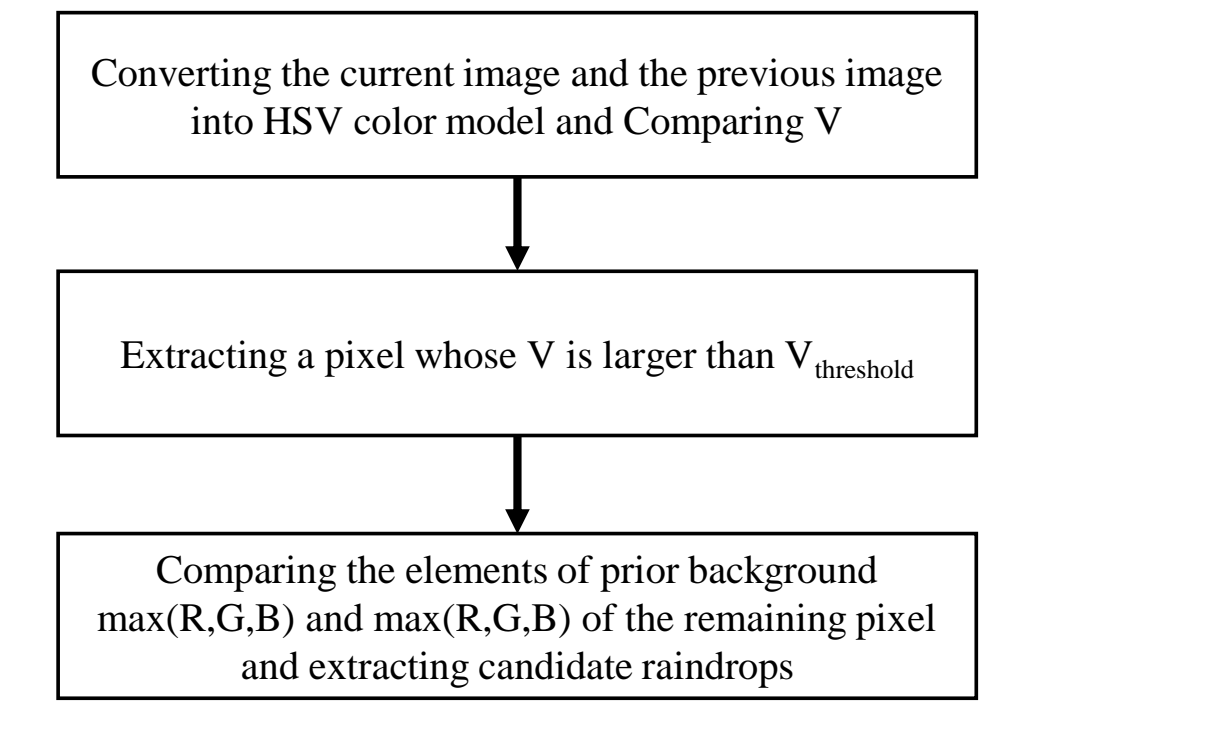

Figure 4. Flow Chart for Extracting Candidate Raindrops

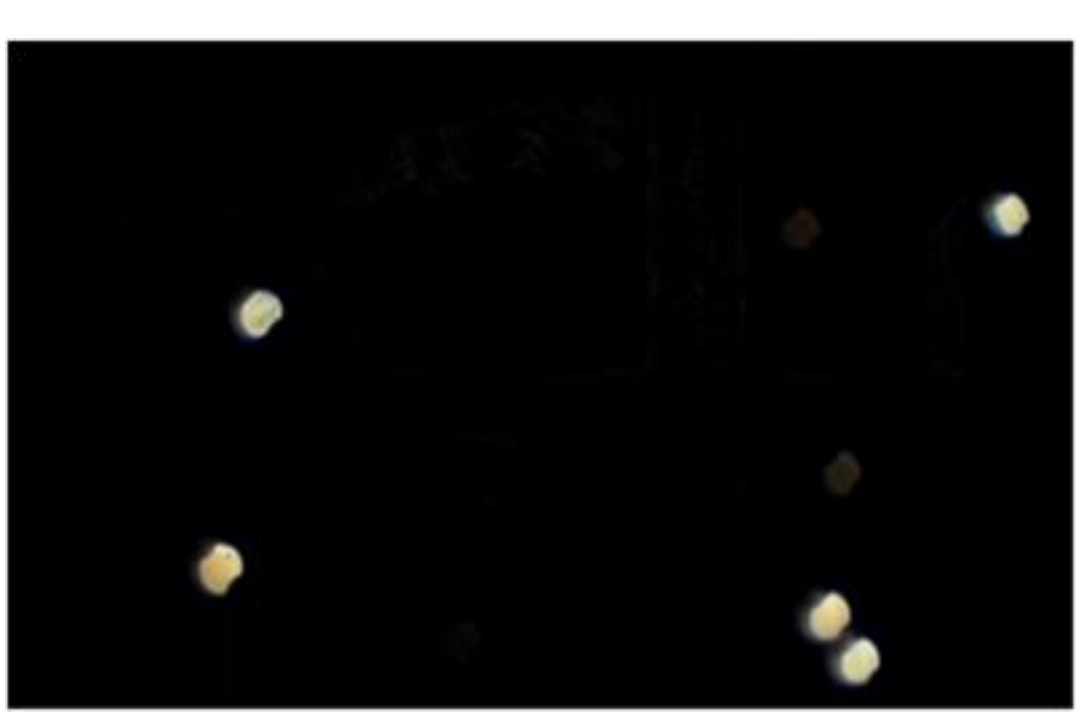

Figure 5. Candidate Raindrops

\subsection{Algorithm to Remove Raindrops}

This section shows the removal of raindrops from images by utilizing the volume of extracted candidate raindrops and predicted raindrops. The dynamic algorithm is applied according to the volume of the predicted raindrops.

\subsubsection{Small volume of Raindrops}

When the volume of raindrops is small, five frames, among which, one frame from the present images and four frames from the previous images are used for removing raindrops. Here, five is an arbitrary number. When the volume of raindrops is small, an algorithm utilizing the largest frequency value is used, and therefore it is good to use five or more images. The use of considerable images decreases the nature of real time, and raindrops are continuously discovered in the selected relevant pixel making it unsuitable. Figure 6 shows brightness and location of raindrops of the relevant pixel selected as candidate raindrops over time. As shown, when raindrops appear in the relevant pixel, the brightness of that pixel 
increases. When raindrops do not appear in the relevant pixel, the brightness is maintained and may change by the appearance of other objects or small light. Therefore, when the volume of raindrops is small, the algorithm for removing raindrops replaces the candidate raindrop pixels with the relevant pixels by calculating the most frequently occurring values of $\mathrm{R}, \mathrm{G}$, and $\mathrm{B}$ among the pixels at the same locations in the current image and in four prior images, that is, in the five images. Nonetheless, there are cases when the most frequent occurring value does not appear and in such cases, information of the fixed background is used. By using the Euclidean distance of R, G, and B of the fixed background and those of the five images, the minimum value is calculated, and raindrops within the image are removed by replacing the relevant pixels.

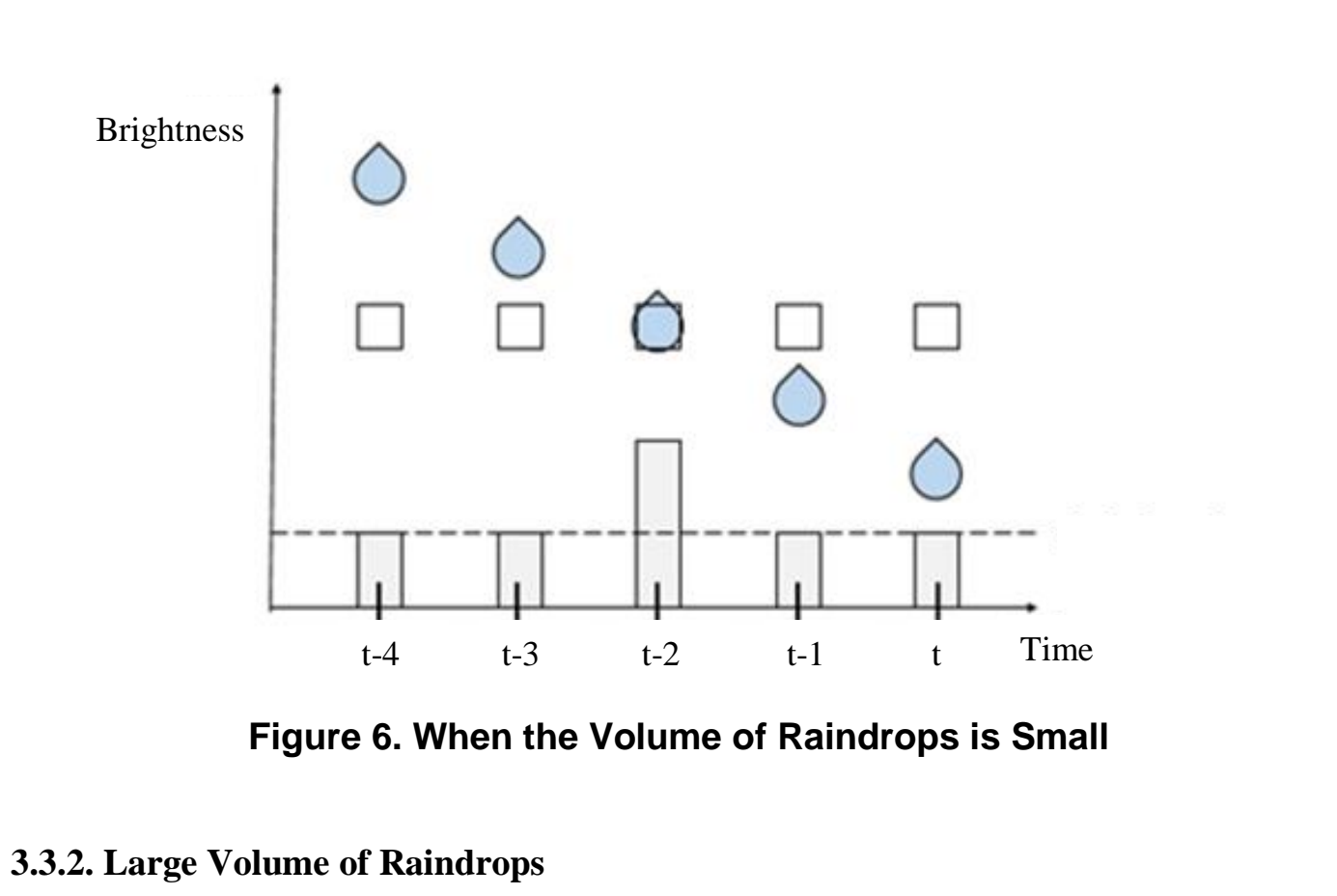

When the volume of raindrops is large, the frequency of raindrops appearing in the pixels increases than then the volume of raindrops is small. Therefore, when the most frequently occurring yalue is used, there might be instances when the pixels are replaced with raindrops rather than the background. Figure 7 shows the situation when the volume of raindrops is large. Moreover, the pixels in such a situation appear frequently. Therefore, pixels whose brightness is lower than the threshold value are selected from among a minimam of 10 images, and the average values of R, G, and B among the pixels is calculated to replace the relevant pixel and remove raindrops. The minimum value is not used because brightness decreases due to the presence of other objects. 


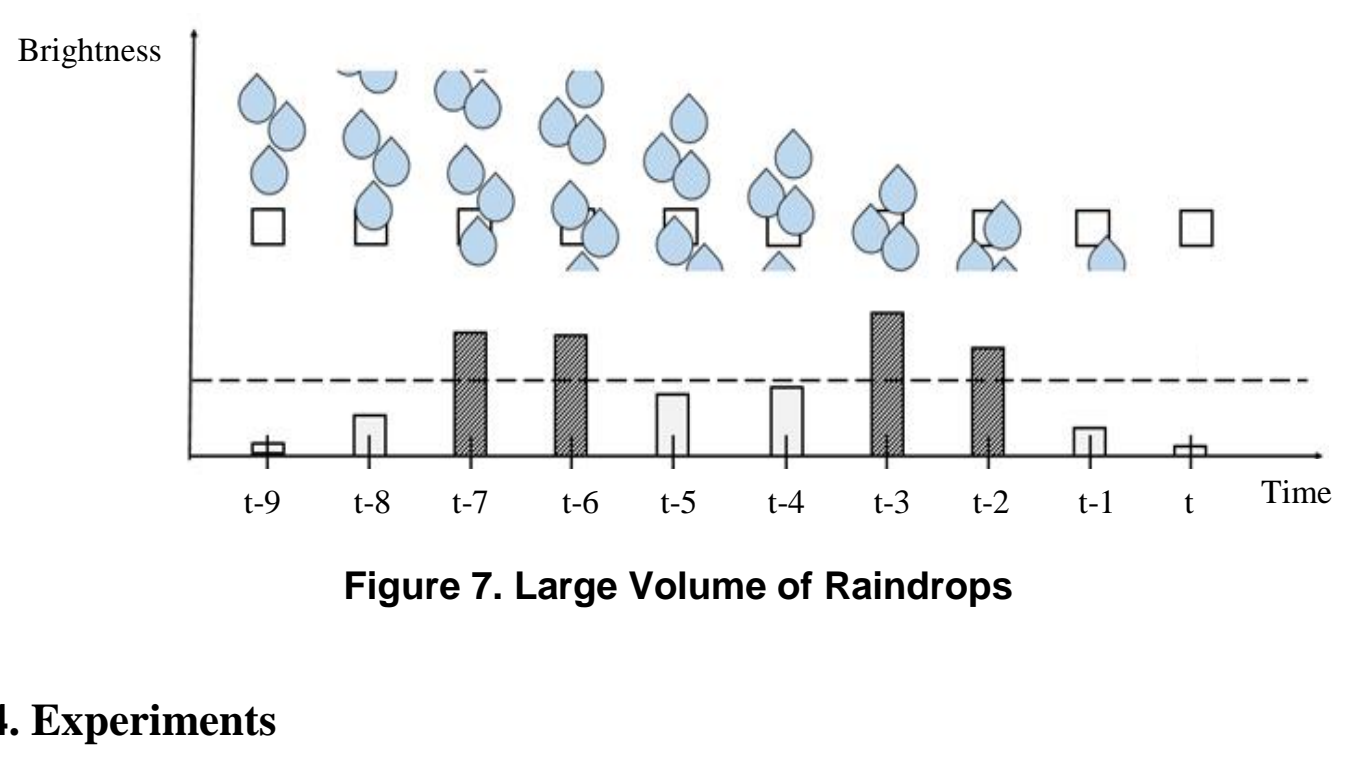

To examine the effect of the algorithm proposed in this papen the actual program was embodied and experimented. Only the case when the volume of raindrops was small was tested because it was impossible to adjust the volume of raindrops or have rain drop.

\subsection{Experimental Setups}

Table 1 shows specifications of the server and camera hardware used in this study.

Table 1. Specifications of the Server and Hardware

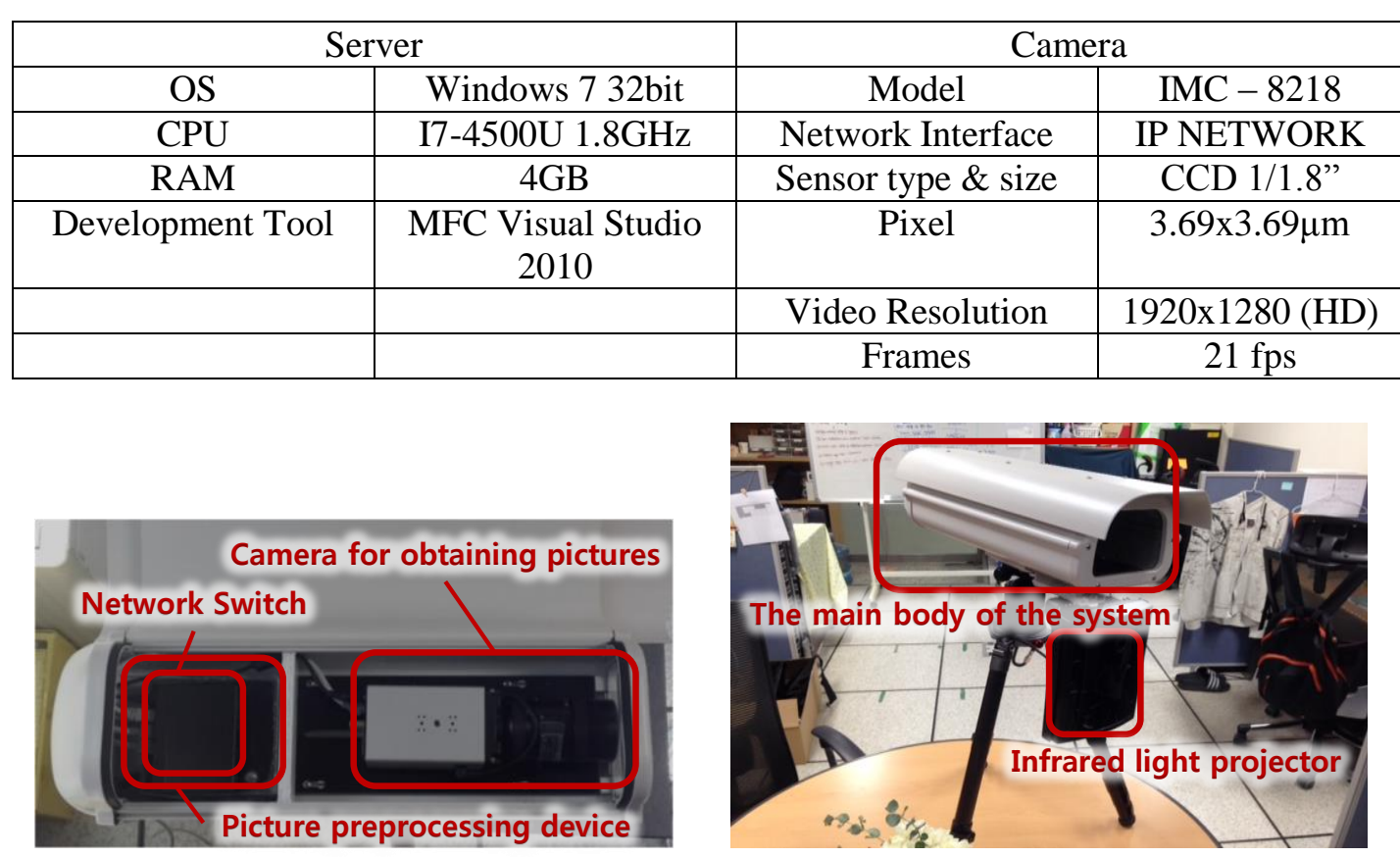

Figure 8. Equipment to Obtain Fixed Pictures

Figure 8 shows the equipment to obtain fixed pictures. This equipment comprises a camera for obtaining pictures, a picture-preprocessing device, and a network switch. The picture-preprocessing device is also used as a server. 
The software for picture processing extracts candidate raindrops through the proposed algorithm from the images obtained at a certain interval by the camera. The camera transmits images to the server at a certain interval through the equipment used for obtaining fixed pictures. The server compares brightness of the transmitted images with that of the preceding images and extracts candidate raindrops by using the color of the fixed background. Next, the volume of the extracted raindrops is determined, and an algorithm to remove raindrops according to the volume of raindrops is dynamically selected.

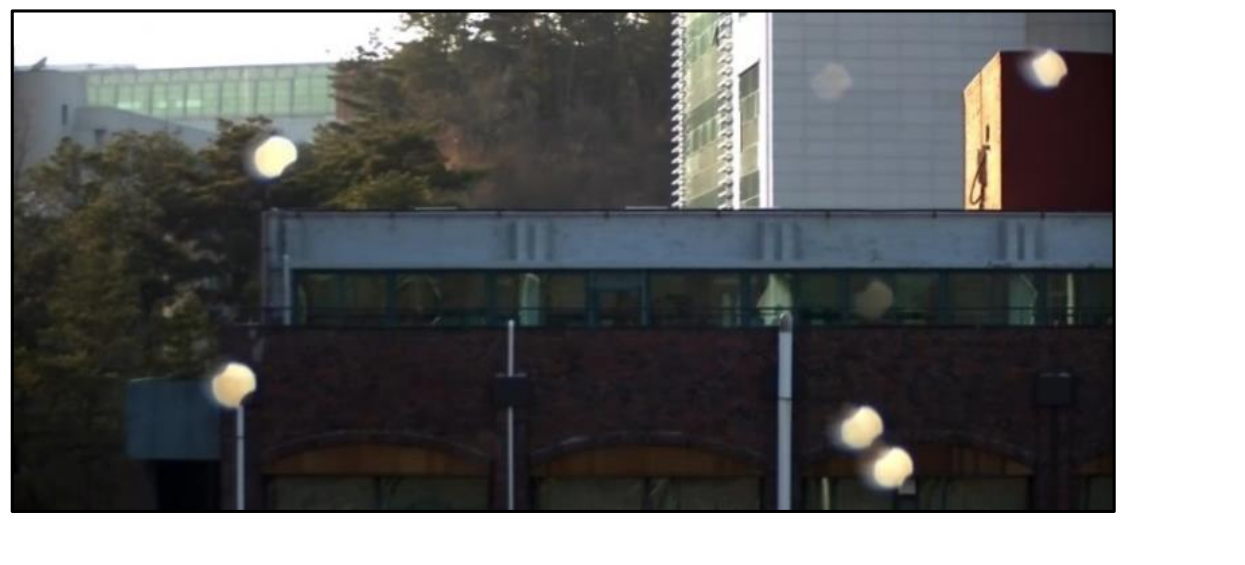

(a) Before Improvement

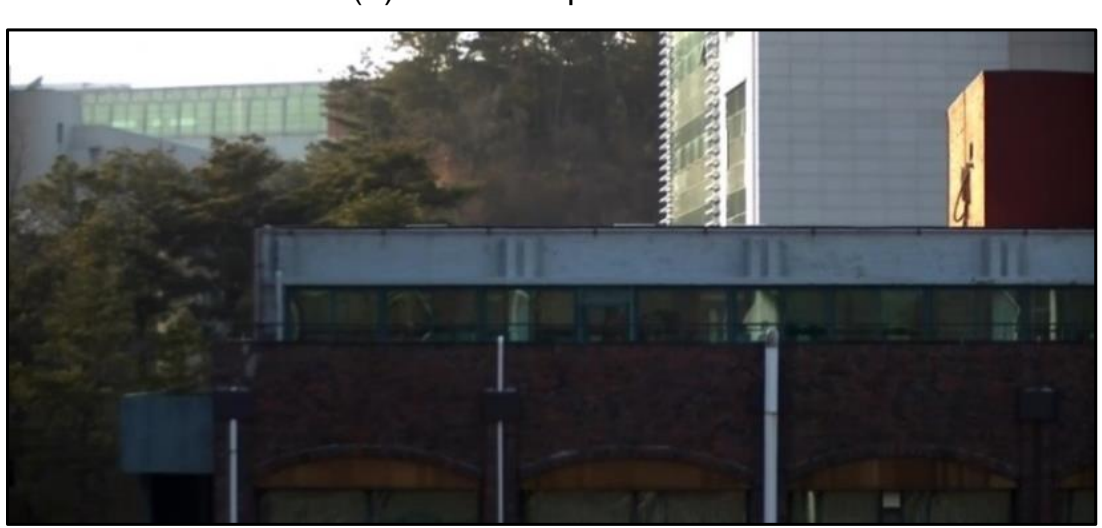

(b) After Improvement

Y Figure 9. The Pictures after Removing Raindrops

\subsection{Experimental Results}

In this experiment, a raindrop removal algorithm was applied when the volume of raindrops was small. Figure 9 shows a picture in which the proposed algorithm was applied and raindrops were removed. The images from which raindrops were not removed shows raindrops obstructing the field of vision of the interested object. After the application of the algorithm, clear images were obtained because there were no raindrops. Figure 10 plots the rate of raindrop removal according to the number of input pictures and the rate of raindrops. In the proposed raindrop removal algorithm, the largest frequency value should be used when the volume of raindrops is small; therefore, the experiment was conducted by inputting an odd number of pictures. The rate of raindrops was varied according to the number of input pictures, and the rate of raindrop removal was measured. When the rate of raindrops was $10 \%$, the rate of raindrop removal was verified to be more than $95 \%$ regardless of the number of input pictures. When the rate of raindrops was $20 \%$ 
and $30 \%$, more than $90 \%$ raindrop removal rate was verified if improvement was made using nine or more images.

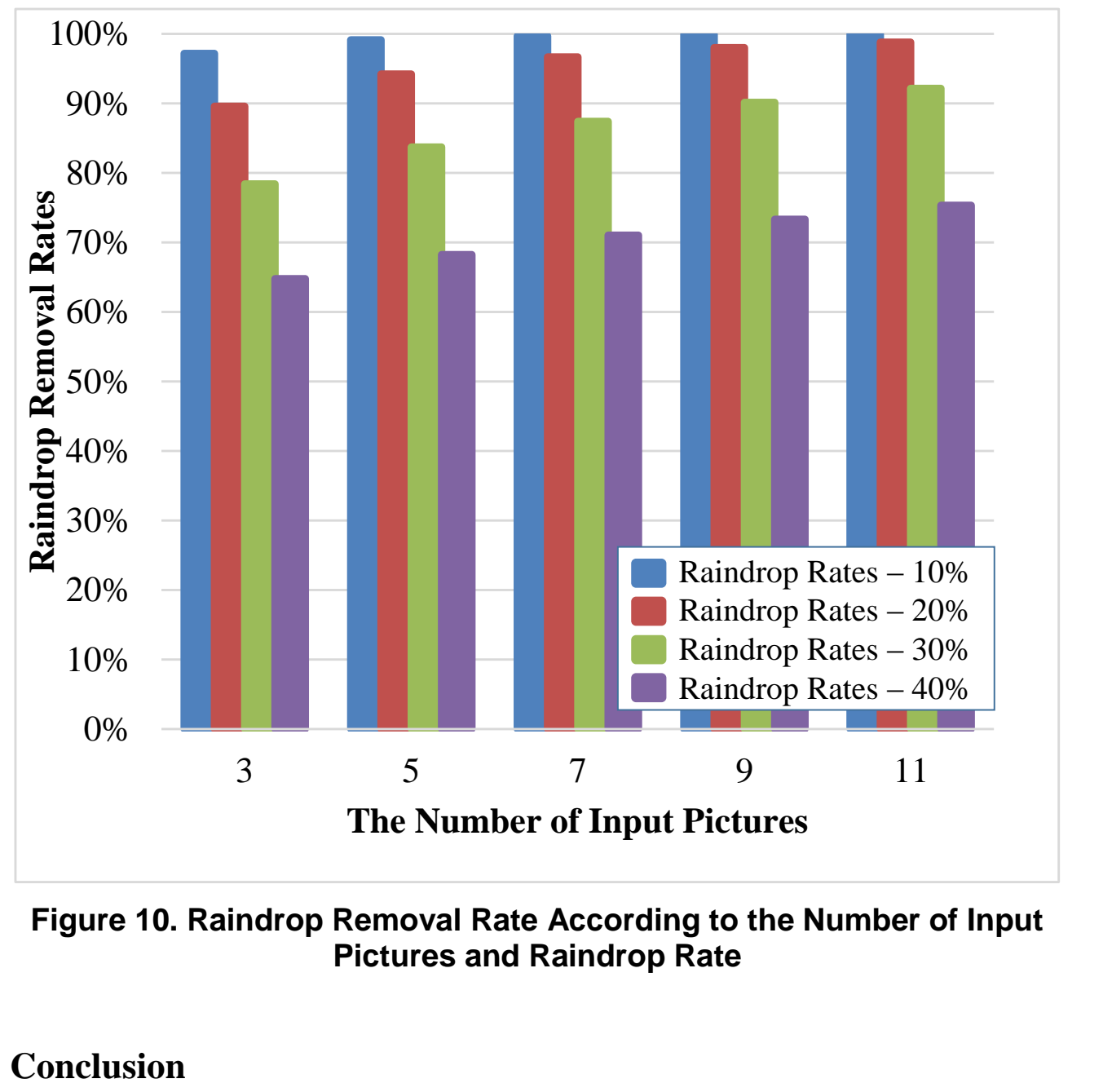

In this paper, a dynamic algorithm is proposed that considers the volume of raindrops and is aimed at removing raindrops within a fixed background video image. In the existing technologies, $\mathrm{R}, \mathrm{G}$, and $\mathrm{B}$ values of a certain image and its earlier version are compared or the angle and size of raindrops are used to extract raindrops. In addition, a model using the color of raindrops and their dynamic characteristics is used to extract and remove raindrops. However, in this study, picture brightness was compared by receiving video images from a camera at certain intervals, the final candidate raindrops were extracted using information on the fixed background, and the volume of raindrops with the volume of candidate raindrops within the pictures was predicted. Further, a dynamic algorithm is proposed, which considers the volume of raindrops. In addition, a program applying the proposed algorithm was developed and tested, showing a better result. The proposed raindrop removal algorithm removes obstacles obstructing information, which is intended to be obtained from pictures and may be helpful for obtaining more precise picture information on the objects. Future research will increase the number of images necessary for efficiently removing raindrops, and a more profound raindrop removal algorithm will be proposed. 


\section{Acknowledgments}

This work was supported by Kyonggi University Research Grant 2015.

\section{References}

[1] HIS, “Top Video Surveiliance Trends for 2016”, HIS blog, (2016).

[2] K. He, J. Sun and X. Tang, "Single Image Haze Removal Using Dark Channel Prior", IEEE Transactions on Pattern Analysis and Machine Intelligence, vol. 33, (2010), pp. 2341-2353.

[3] K. Grag and S. K. Nayar, "Detection and Removal of Rain from Videos", IEEE Computer society Conference on Computer Vision and Pattern Recognition, vol. 1, (2004), pp. 528-535.

[4] S. G. Narasimhan and S. K. Nayar, "Vision and the Atmosphere", International Journal of Computer vision, Vol. 48, (2002), pp. 233-254.

[5] K. V. Beard and C. Chuang, "A New Model for the Equilibrium Shape of Raindrops", Kournal of Atmospheric Science, (1987), pp. 1509-1524.

[6] R. Gunn and G. D. Kinzer, "The Terminal Velocity for Water Droplet in stagnant Air , Journal of Meterology, vol. 6, (1949), pp. 243-248.

[7] R. M Manning, "Stochastic Electromagnetic Image Propagation", (1993).

[8] K. Grag and S. K. Nayar, "When does a camera see rain?", Tenth IEEE International Conference on Computer Vision, Beijing, China, (2005).

[9] W. J. Park and K. H. Lee, "Rain Removal Using Kalnan Filter in Video", IEEE International Conference on Smart Manufacturing Application, Gyeonggi-do, (2008)

[10] X. Zhang and H. Li, "Rain Removal in Video by Combining Temporal and Chromatic Properties", IEEE International Conference on Multimedia and Expo, Toronto, Canada, (2006).

[11] K. Sajitha and D. Venkataraman, "Restoration of Video by Removing Rain", International Journal of Computer Science, (2012), pp. 19.

\section{Authors}

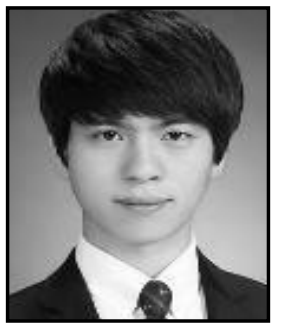

Nambong Ha, received the B.S. degree in Computer Science from the Kyonggi Unversity, Korea, in 2015. He is currently M.S Candidate in Computer Science from Kyonggi University. His research interests include computer vision, software defined network.

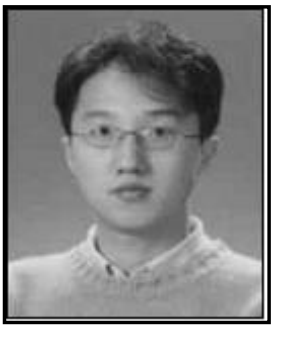

Namgi Kim, received the B.S. degree in Computer Science from Sogang University, Korea, in 1997, and the M.S. degree and the Ph.D. degree in Computer Science from KAIST in 2000 and 2005, respectively. From 2005 to 2007, he was a research member of the Samsung Electronics. Since 2007, he has been a faculty of the Kyonggi University. His research interests include sensor system, wireless system, and mobile communication. 
International Journal of Multimedia and Ubiquitous Engineering

Vol.11, No.8 (2016)

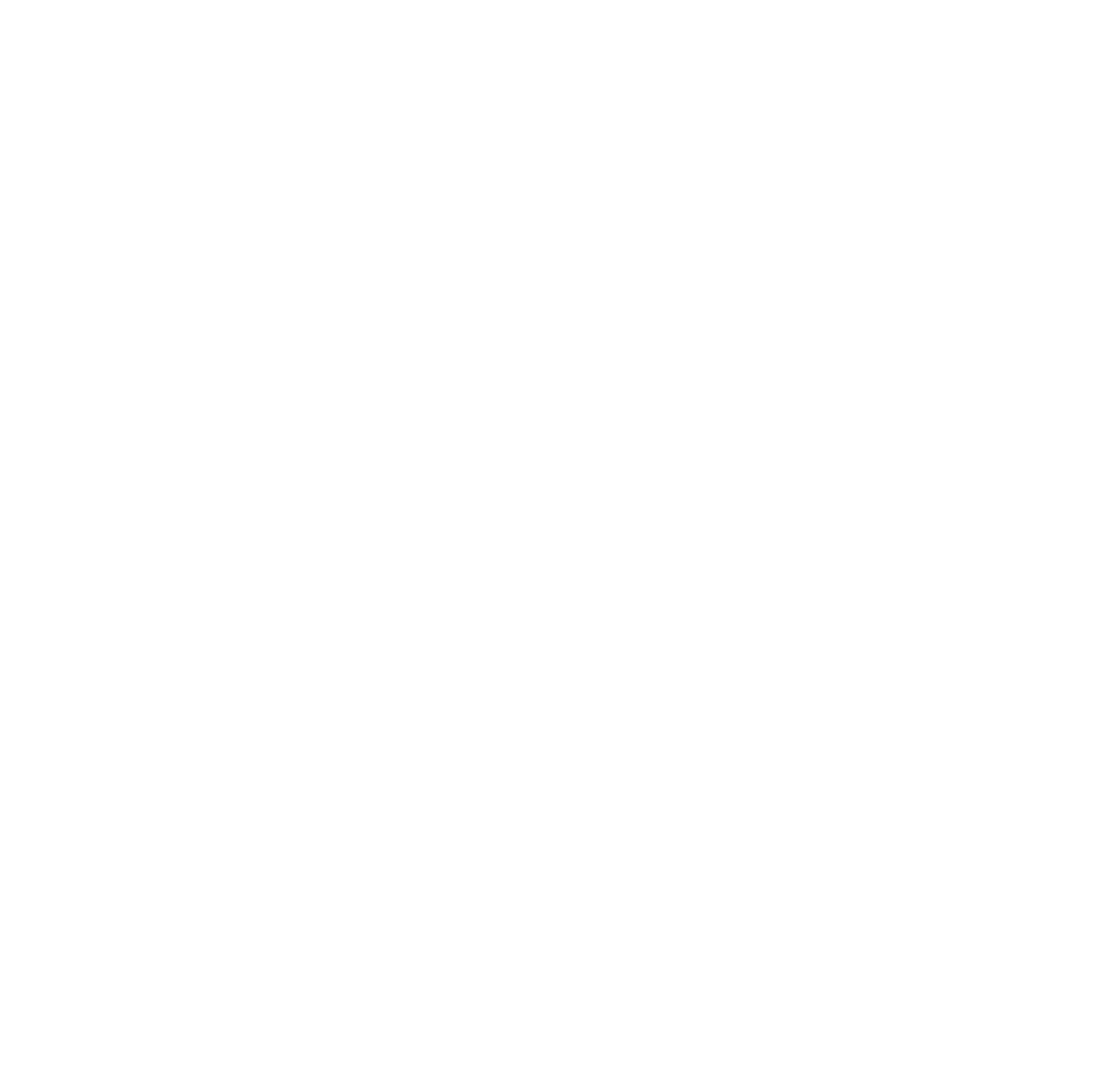

